\title{
La actualidad del pensamiento de Mary E. Richmond en el Trabajo Social italiano
}

\section{The currency of the thought of Mary E. Richmond in Italian Social Work}

\author{
Marilena Dellavalle \\ Dipartimento Scienze Sociali Universidad de Turín \\ marilena.dellavalle@unito.it
}

Recibido: 30/04/2011

Revisado: 06/05/2011

Aceptado: 20/06/2011

Disponible on line: 29/09/2011

\section{Resumen}

En este artículo se analiza la aportación que el pensamiento de Mary Richmond, tal y como se presentó en su libro de 1922 ¿Qué es el trabajo social de caso?, sigue proporcionado al Trabajo Social actual. Tras situar su contribución en el contexto italiano, se apuntarán aquellos aspectos que aún hoy siguen siendo cruciales para la construcción del saber y la acción profesional, en un intento de centrar la atención en aquellos factores que han permanecido y permitido, no siempre explícitamente, que la disciplina haya discurrido por los cauces de continuidad interna de la propia tradición investigadora. De las numerosas sugerencias que emergen del estudio de esta obra, se han escogido las que siguen pareciendo significativas aún hoy, incluso frente a los desafíos que plantea la reducción de los recursos y las directrices de la política social: la posición activa del sujeto en el proceso de ayuda, la centralidad de la persona, la confianza en el cambio y en el papel de los hábitos, la adaptación activa y transformadora, la orientación promocional en el trabajo con los recursos y, por último, las conexiones entre las personas, las condiciones de vida y el sistema de recursos.

Palabras clave: método democrático, centralidad de la persona, promoción, reformas, recursos, potencialidades.

\begin{abstract}
Hereinafter, an analysis of the contribution that Mary E. Richmond's thought, as presented in her 1922 work What is Social Case Work?, may provide nowadays to Social Work. After placing her contribution in the Italian context, aspects of her work considered even now as fundamental in the construction of professional knowledge and action will be identified. Attention will be focused on those factors which have persisted and have permitted, if not always in an explicit way, the discipline to flow within the banks of the internal continuity of a research tradition. Among the many areas of reflection that the text offers, the ones that appear most relevant today will be focused upon, despite the challenges we are experiencing due to the contraction of resources and to the guidelines of social politics, e.g. the active position of the individual in the help process, the centrality of the person, confidence in change and the role habits play, active and transformative adaptation, encouragement in working with resources, and finally, connections between people, living conditions and the system of resources.
\end{abstract}

Keywords: Democratic method, centrality of the person, encouragement, reforms, resources, potential.

Referencia normalizada: Dellavalle, M. (2011). «La actualidad del pensamiento de Mary E. Richmond en el Trabajo Social italiano». Cuadernos de Trabajo Social, 24: 101-111.

Sumario: Introducción. 1. Fragmentos de una herencia: ¿Qué es el Trabajo Social? 2. El proceso de ayuda como método democrático. 3. La personalización y la adaptación activa y transformadora. 4. El trabajo con los recursos y el planteamiento de la promoción. 5. Explorar nuevas posibilidades: entre la confianza en el cambio y la acción rutinaria. 6. Reflexiones finales. 7. Referencias bibliográficas.

\section{Introducción}

En la literatura contemporánea del Trabajo Social italiano, la figura de Mary Richmond ha sido objeto de citas, más o menos extensas, en relación con el papel que desempeñó en la fundación de la disciplina y en la crea- 
ción del método: para Dal Pra Ponticelli sus estudios representan el momento más significativo del «paso del trabajo social como "arte", basado en la disponibilidad e intuición del autor, al trabajo social como "ciencia", fundado en la utilización de unos conocimientos teóricos reconocidos y de un proceso» (1987, p. 117). Según Benvenuti y Gristina (1998), «es una de las figuras fundamentales del trabajo social americano; es más, de todo el trabajo social» (p.77); por su parte Bortoli (2006), considera determinante su contribución a las formas organizativas y de intervención, a la formación y a la investigación, y la considera la «verdadera» fundadora de la ciencia del Trabajo Social (p. 199); asimismo para Fargion (2009), ha llevado a cabo «el primer esfuerzo explícito por convertir en científico el trabajo social» (p. 11), mediante un esfuerzo riguroso de sistematización, cuyos resultados han desembocado en la célebre obra Diagnóstico social.

A pesar de haber sido extensamente citada como la fundadora del Trabajo Social, el pensamiento de Richmond no se ha divulgado en la realidad italiana: en efecto no existe ninguna traducción, ni siquiera parcial, de sus aportaciones, al contrario de lo que ha sucedido en otros países europeos, como Francia o España. Las únicas referencias explícitas y extensas a su pensamiento figuran en el volumen de Tarugi (1961), pero no ha habido otros autores italianos que hayan recuperado posteriormente sus aportaciones, en otros términos que no sean los de la mera cita. Para comprender mejor este vacío hay que hacer una breve referencia al contexto de la segunda postguerra, cuando se consolida el Trabajo Social italiano ante la necesidad, particularmente sentida por el nuevo régimen democrático, de contar con profesionales competentes y oportunamente formados, cuando con lo que se contaba era con la única experiencia de la escuela fascista de asistentes sociales. Jugó un papel crucial, en el camino para introducir de la disciplina en Italia, la Administración de las Ayudas Interna- cionales $^{1}$ que se ocupaba, entre otros, del programa de ayuda financiera y técnica a las escuelas de Trabajo Social en cuyo ámbito se alentó la introducción de los trabajos teóricos estadounidenses, a través de las misiones de expertos extranjeros en Italia y de una intensa actividad de traducción y publicación de la obra de autores americanos ${ }^{2}$. No hay que olvidar que la teoría del Trabajo Social estadounidense, en el momento en que penetró en el contexto italiano, estaba ya sistematizada dentro de nuevas contribuciones, que en parte continuaron y en parte se distanciaron de la orientación de Richmond, cuya obra se consideraba, quizá de momento, demasiado lejana como para añadirla a los textos que era conveniente importar.

Además conviene aclarar que lo que en un primer momento llegó a Italia como aportación de la Escuela Diagnóstica, había surgido de los trabajos contenidos en Diagnóstico social (Richmond, 1917), pero estaba ya profundamente permeada por el Psicoanálisis. Es posible que este maridaje haya provocado el doble equívoco que se ha extendido en el contexto italiano: por un lado, el de considerar la obra de Richmond deudora del Psicoanálisis por su acercamiento al mismo; y por el otro, el de la presunta obstinación de la autora por un trabajo de caso que infravaloraba el componente social. Sin embargo se puede suponer que nuestra autora no se encontraba en esta posición, visto el contenido de esta afirmación suya: «He gastado veinticinco años de mi vida intentando que se acepte el trabajo de caso como procedimiento válido en trabajo social. Ahora agotaré el resto de mi vida en tratar de demostrar a los trabajadores sociales de caso que el trabajo social es mucho más que el trabajo de caso» (citado en Bortoli, 2006, p.196). En ¿Qué es el Trabajo Social de caso? ella misma afirma la dependencia recíproca de las funciones de los trabajadores sociales vinculadas al trabajo de caso, a la intervención colectiva, a la investigación social y a la promoción de las reformas (Richmond, 2006, p.106). Vale la pena

${ }^{1}$ La Administración Ayudas Internacionales (AAI, 1947 -1977) sigue en Italia la actividad de la UNRRA (United Nations Relief and Rehabilitation Administration, 1943-1947).

${ }^{2}$ La bibliografía italiana del Trabajo Social cuenta, en 1966, con 132 títulos traducidos de publicaciones extranjeras. 
recordar que Richmond no sólo ha teorizado acerca de la conexión entre estas diversas funciones, sino que se volcó a la investigación social, desde dentro del departamento que dirigía en la Foundation Russell Sage, y se desvivió para promover reformas legislativas, como la inherente al trabajo de los menores (Bouquet, 2006, p. XII).

En relación a la atribución de una matriz psicoanalítica a su obra, en realidad, como afirma Leonard, Diagnóstico social «no contenía ni una sola referencia a Freud» (1917, p. 13); Bortoli pone también en evidencia la clara preocupación de Richmond, tras el éxito de su libro, por el riesgo que deriva de infravalorar la importancia que desempeñan las condiciones de vida, tanto en el análisis como en la intervención. Según este autor: «el trabajo de Richmond refleja un periodo en el que las ciencias sociales, en particular la sociología, tuvieron una gran influencia en la práctica del trabajo social, mientras la psicología se hallaba todavía en sus primeros pasos» (2002, p. 138); y además su misma fama se vio empañada precisamente en los años veinte, «cuando las nuevas teorías psicológicas hicieron parecer anticuada su perspectiva, al considerarla prevalentemente sociológica» (1997, p. 95). En esta misma dirección, Leonard cita la afirmación de Robinson de que la obra de Richmond define «la fase sociológica del case work que precede a la psicológica de los años veinte-treinta» (Robinson, 1939, citado en Leonard, p. 14).

La importancia que nuestra autora atribuye a los aspectos sociales es subrayada también por Du Ranquet en 1989, cuando resalta como, por influencia de las teorías psicoanalíticas, su posición inicial va sucesivamente cediendo el puesto a otra centrada más en los problemas y los recursos del individuo que en los del contexto.

En ¿Qué es el Trabajo Social de caso? afirma la propia autora-allí donde identifica como fin del Trabajo Social el desarrollo de la personalidad-que su acercamiento al concep- to de personalidad está guiado por las aportaciones de las ciencias sociales ${ }^{3}$. La única referencia a un estudioso de psicoanálisis se encuentra en Diagnóstico social y se refiere a la cita de la afirmación de J.J. Putnam ${ }^{4}$ que resalta la importancia de los factores sociales en el proceso de comprensión de la persona: «en las relaciones sociales de cada hombre es donde se halla escrita su historia mental y en las relaciones sociales es donde deben buscarse principalmente las causas de los desórdenes que amenazan su felicidad y su funcionalidad, además de los medios para asegurar su respuesta» (cit. en Richmond, 1917, p. 4).

\section{Fragmentos de una herencia: ¿Qué es el Trabajo Social de caso?}

En el volumen ¿Qué es el Trabajo Social de caso? -que según Amendola (2002) contiene los principios fundamentales del Trabajo Social profesional- Mary Richmond analiza, a partir de un profundo examen de seis casos tratados mediante la intervención de Trabajo Social con individuos (trabajo de caso), la acción profesional en la que identifica: objetivos, especificidades y contenidos que constante y explícitamente manifiestan valores y teorías de referencia. El fin último del Trabajo Social se extrae de la definición que propone: "conjunto de métodos que desarrollan la personalidad, "reacomodando" entre ellos, consciente e individualmente, a la persona y su contexto social» (Richmond, 2006, p. 48). Cuando precisa el significado que tiene para ella el concepto de personalidad, lo que está indicando es cuáles son los elementos que lo constituyen, es decir, tanto la herencia genética como lo que la persona ha ido adquirendo con la educación, las relaciones sociales y la experiencia (p. 45).

En su forma de tratar el tema afloran elementos muy innovadores, respecto al contexto cultural en el que han sido elaborados y, a la vez, sorprendentes por su actualidad: como afirma Bouquet en la rigurosa introducción a la última edición francesa de la obra,

3 Richmond, 1922, trad fr. 2006, p. 45 n. 4.

4 James Jackson Putnam, (1846-1918), psicoanalista americano -cuyo papel fundamental en la difusión del psicoanálisis en Estados Unidos es reconocido por el propio Freud en el prefacio de la obra de J. J. Putnam (1921). Addresses on Psycho-Analysis-disiente de Freud en lo relativo a la importancia asignada a los factores sociales en el funcionamiento mental de los individuos. 
los motivos que llevan a prestar atención actualmente a las aportaciones de Richmond derivan del hecho de que la exposición de los principales saberes prácticos y metodológicos aportan elementos de reflexión que son pertinentes para el Trabajo Social contemporáneo.

Entre los distintos estímulos -en estrecha relación con la última edición francesa de este libro (Richmond, 2006)- proponemos aquellos que son inherentes al respeto de la persona como ser único e irrepetible y como sujeto activo y reflexivo; a la consideración de la conexión entre el individuo y el contexto; a la orientación de la promoción del trabajo con los recursos; al acercamiento democrático en un proceso de ayuda orientado a promover la reflexión y a sostener la investigación de nuevos itinerarios para afrontar tareas y problemas existenciales; $y$, además, a la vinculación entre el trabajo individual y la promoción de las reformas.

Amendola opina que Richmond basa la disciplina del Trabajo Social en la «concepción de la persona como ser inteligente, perfectible, introducido en un contexto real en el que están también insertados los obstáculos materiales a su desarrollo: el eje lo fija la capacidad humana de reflexionar y de pensar en tales circunstancias y de imaginar cambios futuros posibles» (2002, p. 175). En opinión de esta autora, este posicionamiento se diferencia claramente de la postura compasiva, se funda en la confianza en las capacidades del Otro y en la promoción de dicha potencialidad, a partir de la consideración del sujeto como «protagonista y generador del propio proceso de cambio», proceso que inicia precisamente en el momento en que se reconocen los obstáculos y los factores -individuales y sociales-que influyen en las circunstancias de la vida (ibidem).

También la elección de Richmond, cuando se refiere a diversas áreas disciplinarias, como la Sociología, la naciente Psicología social o la Filosofía ${ }^{5}$, nos permite identificar una primera conexión con el debate contemporáneo acerca del proceso de construcción teórica en Trabajo Social: en efecto, creemos po- der asegurar que en su propuesta teórica se encuentra aquella multiplicidad epistemológica de referencias, intrínseca a la naturaleza del Trabajo Social, que responde a su «necesidad de remitirse también en el plano hermenéutico a una pluralidad de referencias» (Gui, p. 31) para dirigir la atención contextual a la persona, al tejido social y a las formas institucionales de ayuda.

\section{El proceso de ayuda como método de- mocrático}

La relación profesional propuesta por Richmond tiene como objetivo desarrollar la personalidad reflexiva,

A través de un proceso de estimulación de las capacidades conscientes y no por medio del adiestramiento o por la sugestión de las emociones, la obediencia o la gratitud típicas de la beneficencia. La promoción de la reflexión en el otro implica la confianza en el otro como persona (Amendola, 2002, p. 173).

Por esta razón, su perspectiva -al valorar las propiedades de transformación del Otro, percibido como sujeto de derechos- se distancia claramente de las visiones filantrópicas centradas en las carencias y en la posición de debilidad de quien demanda ayuda (ibidem).

Al afirmar que «de todos los sistemas para desarrollar la mentalidad y las relaciones sociales de un cliente, el más eficaz es el de hacerle tomar parte activa», Richmond (2006, p. 42) orienta claramente la intervención del trabajador social a facilitar la participación del sujeto en todas las fases del proceso de ayuda, elemento que, según su expreso parecer, caracteriza el método de intervención profesional como democrático: «el método, mediante el cual se alcanza la comprensión del cliente y la elaboración -en interacción con él- de un programa en el que va a participar, es esencialmente un método democrático» (p. 82).

En lo que denomina programa participativo de acción, cliente y trabajador social comparten la responsabilidad de las acciones que se suceden en el camino hacia el resulta-

${ }^{5}$ Las referencias en esta obra son a: G.H. Mead, H. James, J.M. Baldwuin, J. Dewey, R. Park, E.W. Burgess, W. Lippmann, E.A. Ross, A.G. Heats y F. Adler. 
do esperado; este último constituye para ambos actores un horizonte -como le gusta definirlo (p. 81)- que puede ser identificado como una meta compartida.

Se puede detectar una posible conexión entre la posición de Richmond -referente a prestar apoyo, a encontrar un horizonte para las personas en dificultad que puedan haber abandonado su inversión en el futuro-y la de Pittaluga (2000), que sostiene que mientras para algunas personas las experiencias parecen reforzar un tipo de preferencias que se pueden alcanzar con los vínculos existentes, para otras, en cambio, hay una parte activa y constructiva que trata de abrir nuevos frentes, modificando tanto los vínculos externos como las propias inclinaciones. Según Richmond, es esta segunda posibilidad la que debe promover el Trabajo Social, al generar nuevas oportunidades, al acompañar a la persona en el proceso de imaginar las consecuencias de sus propias elecciones, pero también en el momento de buscar alternativas, nuevos significados y horizontes, teniendo siempre presente -sigue exhortando- como valor de referencia esencial «el respeto a la personalidad, respeto que implica una visión democrática» (p. 81).

\section{La personalización y la adaptación ac- tiva y transformadora}

La obra de Richmond inicia su andadura con su experiencia como responsable de las actividades de la Charity Organisation Society (COS), realidad en la que se plantean sin duda las bases de la filantropía científica y, posteriormente, del Trabajo Social profesional; pero la filosofia de fondo de las COS no se había liberado del concepto clasificador de los pobres como merecedores o no merecedores (Geremek, 1995). Esta tendencia a la clasificación se halla en la afirmación siguiente de un dirigente de las primeras COS estadounidenses: «los casos se dividirían naturalmente en grupos principales, lo que facilita una clara aplicación de la justa ley para cada uno de ellos» (citado en Richmond, 2006, p. 74). Richmond se aleja radicalmente de esta posición, cuando sostiene que ningún programa puede tener éxito si se basa en estos presupuestos y, desde su observatorio de supervisión controla como, a lo largo de la evolución del Trabajo Social, se ha ido abandonando gradualmente esta perspectiva homogeneizadora, sustituyéndola por otra más atenta a las características peculiares de cada historia, cuya base es la reflexión acerca de que las personas que se hallan en la misma situación «no son tan iguales como lo parecen» (pp. 73-74).

$\mathrm{Su}$ reiterada invitación a los trabajadores sociales a que descubran y liberen lo que hay de mejor y único en cada individuo pone las bases de lo que será un elemento permanente en el proceso de evolución del Trabajo Social y que, posteriormente, se sistematizará como uno de los principios operativos de la profesión: el de la individualización y de la centralidad de la persona. Neve (2008), invita a no utilizar ambos términos como intercambiables, ya que desde la perspectiva semántica conviene establecer una diferencia: el primer concepto significa la adecuación necesaria de las respuestas estandarizadas a la situación específica de cada persona, mientras que el segundo hace referencia a la exigencia deontológica de no cosificar a las personas, de respetar su dignidad y de considerarlas como sujetos activos, dotados de intencionalidad (p. 192).

Este principio deriva, por tanto, del valor de la persona en su unicidad, al que debe orientarse axiológicamente la acción profesional, como se refleja en los códigos deontológicos de la profesión en diversos países (Banks, trad. it. 1999) y como lo contempla el propio Código Deontológico italiano:

La profesión se fundamenta (...) en la unicidad de todas las personas (...). El trabajador social (...) considera y acoge a cualquier persona portadora de una demanda, de una necesidad, de un problema como única y distinta de otras en situaciones análogas (CNOAS, 2009, arts. 5-7).

La postura asumida por Richmond en la relación entre individuo y ambiente social es tributaria, como apunta la propia autora, de las aportaciones todavía inéditas de Mead (2006, p. 61): la personalidad-que el Trabajo Social se propone desarrollar frente a condiciones de dificultad-tiene su origen en la sociabilidad, dado que sociabilidad e individualidad no son más que dos aspectos de una 
misma realidad. De esta concepción es de donde deriva el doble y simultáneo ámbito de intervención del trabajador social: la persona del usuario y el contexto social. Este último puede ser modificado también gracias a la acción del trabajador social competente en promover la utilización de los recursos, en relevar o no la congruencia de estos últimos con las necesidades emergentes, en potenciar la creación de nuevas oportunidades, pero también en recurrir a ambientes alternativos si el de origen es «activamente antisocial» (pp. 52-54).

La intervención del trabajador social gira, por lo tanto, en torno a una doble tarea -la comprensión y la acción participativa-que no debe involucrar sólo a la persona del cliente sino también al contexto social en el que vive el sujeto, considerado en términos de recursos, vínculos, riesgos e influencias. De este modo, nos hallamos ante una complejidad cada vez mayor en la que se cruzan cuatro elementos constituyentes -el binomio de la intervención (comprensión y acción) y el de la dimensión de la propia intervención (individuo y contexto social) - que mantendrán una continuidad interna en la evolución del Trabajo Social, aunque sea ocupando posiciones diversas, ya sea de fondo como en un primer plano, en los diferentes momentos históricos y no siempre consigan ser operativas.

Esta doble dimensión del trabajo profesional la retoma Richmond cuando trata el tema de la doble comprensión que ha garantizarse en la acción con la persona y el ambiente: «El trabajador social conocerá una parte importante de la vida de su cliente y comprenderá mejor las dificultades que le atormentan y sus potencialidades innatas cuando haya conseguido hacerse una idea clara de sus relaciones sociales»(p. 62). Nuestra autora, además de deplorar el estilo de intervención que considera a la persona o a la familia como "náufragos en una isla desierta» e infravalora las historias de vida, los intereses, lazos naturales y recursos, anima a no centrarse en los problemas, sino a «acercarse al cliente a través de los aspectos de normalidad que han caracterizado su vida» (p. 64). Una invitación a no demoler sus potencialidades, presente en el pensamiento actual de Ferrario (2004), cuando señala que la tendencia a resaltar ante todo las dificultades y las carencias puede conducir a invisibilizar los recursos (p. 110).

El Trabajo Social debería promover, en la relación entre individuo y ambiente social, un proceso de adaptación que Richmond define «consciente y comprensivo» o "adaptación mutua», dentro del cual no se ha de adaptar forzosamente la persona a su contexto, sino que ha de ser este último el que se modifique (p. 55). Podemos relacionar este concepto con el de «adaptación activa y creativa», elaborado por Germain y Gittermann (trad. it. 1985, p. 164), entendido como «proceso mediante el cual los seres humanos modifican sus propias condiciones ambientales para adaptarlas a sus necesidades y aspiraciones y, viceversa, se transforman activamente ellos mismos para adecuarse a las exigencias aceptables o inmutables del ambiente». Su modelo existencial, introducido en Italia por Dal Pra Ponticelli (1985a), concuerda profundamente con el trabajo realizado por Ferrario, orientado igualmente por la perspectiva ecológica. Este autor rechaza el determinismo genético, psíquico y ambiental y presupone que la personalidad humana sigue desarrollándose a lo largo de todo el ciclo vital, a condición de que interactúe con el ambiente que los autores denominan «nutritivo», es decir, capaz de alimentar su desarrollo, con recursos, estímulos, apoyos y oportunidades.

El concepto de adaptación, así concebido, plantea un claro contraste con el que -en el curso de la contestación de finales de los años sesenta- fue objeto de ásperas críticas: la adaptación de cuño funcionalista, que pretendía favorecer en términos conservadores, la acomodación pasiva de las personas usuarias a los roles sociales para asegurar una reproducción ordenada de la estructura social.

\section{El trabajo con los recursos y el plantea- miento de la promoción}

A propósito de la acción con el contexto social, que definía como indirecta, Richmond pone en evidencia el trabajo con los recursos, entre los que incluye también los servicios que operan en diversos sectores. La función de conexión reticular, que actualmente desempeñan los trabajadores sociales y les es reconocida por la literatura (Ferrario), está ya 
presente en su trabajo, cuando define a este profesional como un «agente de vínculos que trata de utilizar con inteligencia los resursos sociales organizados por el barrio y por la colectividad» (p. 44). Se apresura a señalar, en relación con este elemento, el riesgo de su desnaturalización -muy presente todavía hoy y que debía ya preocupar a los trabajadores sociales de la época- de reducir la intervención a un mero dispositivo de conexión entre personas y prestaciones asistenciales, asumiéndolo como fin en vez de como medio de la propia actuación profesional. La invitación de Richmond a «usar el ingenio para suplir la falta de recursos» (p. 54) parece compatibile con una visión creativa y promotora de la intervención profesional, bastante alejada de la insidiosa orientación burocrática y asistencialista, con que se ha representado constantemente la historia del Trabajo Social.

También el modelo integrado -elaborado por los estadounidenses Alien Pincus y Anne Minahan (1977, trad. it 1985)- ha obtenido notable crédito en el Trabajo Social italiano, siempre gracias a la difusión llevada a cabo por Dal Pra Ponticelli. Este hecho le otorga una importancia estratégica al trabajo con los recursos, al identificar el baricentro de la práctica del Trabajo Social en las interacciones entre las personas y los sistemas de recursos; considerados estos últimos cruciales para el desempeño de las tareas existenciales, pero que pueden resultar inadecuados por motivos como su escasa visibilidad o accesibilidad, sus carencias o una oferta desfasada. Estos autores aportan una interpretación interesante, por lo que se refiere a la accesibilidad: según su opinión, también puede disminuir su valor por la propia definición que las personas dan de esos recursos, definición que les haría dudar a la hora de pedir ayuda o que incluso les podría llevar a eludir a toda costa el contrato. Actualmente, se pueden poner algunos ejemplos significativos en relación a los servicios de salud mental para adultos y menores, y a los servicios destinados a la tutela del menor: muchos ciudadanos rechazan la oferta, en términos explícitos o latentes, de establecer contacto con los primeros ya que, en su representación, cualquier referencia al malestar psíquico se asocia a la locura y, por tanto, al prejuicio respecto a ésta que arrastra consigo la desconfianza por los espacios destinados a su cuidado, y la necesidad de evitar cualquier forma de identificación con aqué1la: mientras los segundos son objeto de representaciones sociales cargadas de temor que hacen alejarse a los padres que se hallan en estas dificultades. Para comprender mejor estas posiciones, es útil recurrir a las contribuciones del interaccionismo simbólico y, en particular, al pensamiento de Blumer (1969, trad. it. 2008), para quien los individuos actúan en relación a las personas y a las cosas de acuerdo con los significados que éstas han asumido para ellos, sin que este hecho lleve a posiciones inmodificables, ya que al ser cada situación potencialmente reinterpretable, se pueden introducir nuevas informaciones que permitan someterla a un proceso de resignificación posterior. Esta perspectiva ha sido criticada por los teóricos de la categorización, en relación a que las «minorías y los marginados están íntimamente unidos a carreras desaventajadas porque los otros son capaces de imponer definiciones acerca de ellos» (Collins, 1988, trad. it. 2006 , p. 279). La conciencia de este hecho aumenta aún más la implicación del Trabajo Social en el acompañamiento a las personas en el proceso de adquisición de las informaciones, y también de sus propios derechos y su exigibilidad dentro del sistema de recursos que le incrementen su capacidad reflexiva. La relación de ayuda, al introducir elementos nuevos en la cadena de interacción del sujeto, puede incrementar el capital cultural y social y la energía emocional, al favorecer la posibilidad de desafiar a la rutina, que se ha demostrado ineficaz para hacer frente al problema (p. 280). Nos parece que estas aportaciones pueden sostener la propuesta de Pincus y Minahan (p. 249) acerca de la tarea que compete al trabajador social como profesional encargado de «establecer los vínculos iniciales entre las personas y los sistemas de recursos» y «facilitar para ello la interacción», introduciendo nuevas informaciones que abran el camino a nuevas definiciones.

Es de gran relevancia la previsión -aún permaneciendo como idea de fondo, pero sin que sea posible expresarla como una realidad 
operativa constante- de lo que Pincus y Minahan (p. 251) definen como la función de contribución al desarrollo y al cambio de la política social, llamando la atención sobre las necesidades aún insatisfechas, sobre los vacíos existentes en los recursos actuales y sobre los aspectos disfuncionales de la política y de la legislación social. Una previsión que, consideramos, se puede encontrar en la invitación que Richmond dirige a los trabajadores sociales, a: «demostrar la propia devoción al bien público ejerciendo presiones para que se creen las condiciones para colmar las lagunas descubiertas» (p. 54). Para ejercer dichas presiones, los trabajadores sociales han de utilizar todos los argumentos posibles, derivados del trabajo con cada caso, que podrán ser casos probados respecto a la necesidad de activar nuevos recursos. De este modo la autora resalta el vínculo entre el trabajo de caso y el programa de acción social más amplio. $\mathrm{Su}$ propuesta es que estos profesionales se conviertan en «testigos fieles» de la necesidad de reformas sociales, necesidad que surge de lo que sucede en el trabajo con las personas (p. 106).

\section{Explorar nuevas posibilidades: entre la confianza en el cambio y la acción rutinaria} «La mentalidad, esa mentalidad humana no es fija ni inalterable (...) por el contrario vive, crece, cambia (...), es capaz de recibir potentes estímulos del exterior, de formar nuevos hábitos, de aprovechar las ocasiones que se presenta» (Richmond, 2006, p.61). Con esta afirmación la autora parece querer representar de modo inequívoco la confianza en el cambio, concepto que orienta en términos explícitos a un replanteamiento teórico del Trabajo Social $^{6}$. Su propuesta se basa en la visión del individuo quien, ante las necesidades y los obstáculos, usa las fórmulas del razonamiento y recurre a los hábitos para salir del círculo de las reacciones instintivas y entrar en la espiral donde se suceden nuevas combinaciones ( $p$. 79), y abren nuevos horizontes: un individuo que ella misma compara con un explorador. Se nota aquí la influencia de los pragmáticos, en relación concretamente con la excepcional capacidad humana de crearse hábitos, con la conciencia desarrollada del Yo que actúa en el mundo exterior y con el pensamiento como actividad renovadora (Dewey, 1925).

A propósito de los hábitos, parece interesante resaltar la conexión con uno de los presupuestos de la teoría voluntarista de la acción: el de la tipificación (Marletti, 2006); concepto que se refiere al proceso cognitivo que fija en la mente los rasgos comunes de una situación o de un curso de acción, separando los aspectos contingentes y variables de los que parecen reproducibles, para poderlos reconocer y recordar cuando sea necesario. Al no considerar correcto asimilar los modelos de comportamiento como las modalidades de actuación mecánica y repetitiva y para explicar la diferencia, este autor recurre a la eficaz metáfora de la representación teatral o de la ejecución musical, en donde tiene lugar no una repetición sino más bien una reinterpretación creativa (op. cit.).

Según Berger y Luckmann (1969), la habituación libera al actor de la necesidad de tener que redefinir cada situación desde el inicio, le permite prever mentalmente las actividades que ha de llevar a cabo y hace posible una valoración acerca de las diversas alternativas de comportamiento, liberando así nuevas energías de elegibilidad e innovación (pp. 82-83). La idea del individuo que participa en la formación de nuevos itinerarios es ya nuclear en la perspectiva de Richmond, hasta tal punto que le hace concebir la acción profesional del trabajador social como dirigida a descubrir nuevos significados y posibilidades en las situaciones $\mathrm{y}$ nuevas maneras de actuar mediante la introducción de distintos estímulos en las mentalidades debilitadas por las circunstancias (Richmond, 2006, p. 48).

En lo que se presenta como continuidad cultural, encontramos la afirmación de Odi-

${ }^{6}$ El modelo existencial de Germain C. y Gittermann A., presupone una concepción del hombre dotado de potencial de crecimiento, desarrollo y aprendizaje que permanece en el curso de la vida. Sobre la confianza en las potencialidades humanas de progresar, véanse también Diomede Canevini (1987) y Ferrario (2004). En relación al cambio como ruta de intervención en Trabajo Social, véase M. Dal Pra Ponticelli (1983). 
le Vallin ${ }^{7}$ (1947), en su intervención en el Congreso «Estudios de Trabajo Social», que tuvo lugar en Tremezzo, en una Italia muy marcada por los valores democráticos más elementales de la reciente lucha por la liberación del régimen nazifascista: «El trabajador social no domestica a la persona», sino que trata de «suscitar sus energías y su espíritu de iniciativa» (p. 747).

Una relación de ayuda con este tipo de orientación puede vincularse con las aportaciones de la Psicología y de la Sociología del Yo, en concreto, con el concepto de self activo, creativo y, sobre todo, reflexivo de Blumer (op. cit.), para quien el individuo no se limita a proporcionar respuestas determinadas por el juego de los factores que confluyen hacia él o en él: es ese vasto proceso de interacción lo que le implica consigo mismo y con los demás.

El hecho de atribuir al proceso de ayuda el objetivo de promover y sostener nuevas vías para afrontar tareas esenciales se conecta también con el concepto del pensamiento como actividad renovadora de Dewey (1925), cuando indica cómo ha influido el proceso de teorización del Trabajo social, a partir del trabajo de Richmond, para continuar en términos significativos con el de Perlman (1962). Una continuidad con esta perspectiva se puede encontrar en la producción del Trabajo Social italiano contemporáneo: Maria Dal Pra Ponticelli representa, en varios momentos, la aportación importante que la teoría del Trabajo Social ha extraído del pensamiento de Dewey, sobre todo por lo que atañe al esquema lógico que subyace en la deficinión del procedimiento metodológico.

\section{Reflexiones finales}

La contribución que el Trabajo Social puede extraer de esta obra de Richmond es mucho más amplia de lo que figura en estas reflexiones. Entre los aspectos, quizá menos conocidos pero indudablemente dignos de atención, se encuentra la concepción que sintoniza con la perspectiva de la trifocalidad o muldimensionalidad (Gui, 2004; Sicora, 2005) que, por lo menos desde el punto de vista teórico, ca- racteriza al Trabajo Social italiano actual; éste prevé el entrelazamiento sistémico de la acción de ayuda a la persona con el contexto social y con la organización en la que opera en trabajador social. Nos referimos a la sugerencia de Richmond de mantener lazos estrechos entre las diversas dimensiones de la acción profesional, en lo que se ha dado en llamar una doble tarea: no cerrarse en lo que se tiene a disposición o en lo que falta, sino promover la aparición de nuevas oportunidades, señalando a los sujetos decididos los recursos que han de activar para evitar escisiones entre necesidades y recursos. A estos deberes le añade un tercero - promover las reformas y, posteriormente, actuar para hacerlas factibles-proponiendo, así, un rol de advocay, vinculado no sólo a la tutela de los derechos de cada individuo, sino también a la promoción de las condiciones capaces de garantizar su exigibilidad. En esta imagen está comprendida también la vinculación del Trabajo Social con la investigación social: de la experiencia de trabajo con los casos individuales, correctamente documentada, los trabajadores sociales pueden ofrecer una base para los estudios de los fenómenos sociales, así como también elaborar modelos operativos bajo la guía de los logros positivos de las intervenciones (Richmond, 2006, pp. 18 y 107).

En el tejer la malla entre los conceptos elaborados por Richmond en los años 1920 y algunas de las tendencias actuales del Trabajo Social, hemos tratado de contribuir a la reflexión sobre el tema de la construcción y de la sedimentación de las bases culturales del Trabajo Social italiano, en su interacción con el internacional. Nos hemos detenido en aquellos elementos que no sólo siguen persistiendo, sino que parecen significativos en su estado actual de elaboración de este saber: somos de la opinión de que, como tales, pueden sostener la hipótesis de una tradición de investigación (Gui, 2004) que constituya la especificidad de la disciplina.

Entre los numerosos elementos del texto en apoyo de esta hipótesis, hemos elegido los que consideramos más cercanos al debate cultural, pero también a los desafíos que se

7 Odile Vallin dirige la primera escuela italiana para trabajadores sociales, surgida en la clandestinidad en el otoño de 1945, durante la segunda guerra mundial. 
le plantean al Trabajo Social, ya sea por la complejidad social o por las directivas de la política social, orientadas por la perspectiva del gerencialismo, que puede inducir a procesos de burocratización capaces de desnaturalizar el mandato profesional. En definitiva, queremos aceptar la invitación de nuestra progenitora a cuestionar incesantemente el pensamiento y la acción sobre el profundo sentido de la presencia del Trabajo Social y de los logros que se propone alcanzar, dentro del actual orden social y frente a las provocaciones de la postmodernidad (p. 60).

\section{Referencias bibliográficas}

Amendola, Viviane (2002). La persona come oggetto dell'azione professionale in Mary Richmond. En C. Marzotto (coord.), Per un'epistemologia del Servizio sociale. La posizione del soggetto (pp. 171-179) Milano: Franco Angeli.

Banks, Sarah (1995). Ethics and Values in Social Work. Londres: British Association of Social Workers, Macmillan, Press LDT. Trad. it. 1999: Etica e valori nel Servizio Sociale. Trento: Erikson.

Benvenuti, Pierangela y Gristina, Domenica (1998). La donna e il servizio sociale. Milano: Franco Angeli.

Berger, Peter L. y Luckmann, Thomas (1966). The Social Construction of Reality. Garden City, Nueva York: Doubleday and Co. Trad. it. 1997: La realtà come costruzione sociale. Bologna: Il Mulino.

Bianchi, Elisa et al. (1983). Servizio sociale, sociologia, psicologia: ripresa critica di un dibattito teorico. Padova: Fondazione Zancan.

Blumer, Herbert (1969). Symbolic Interazionism. Perspective and Method. Englewood Cliffs, NJ: Prentice-Hall. Trad. it 2008: Interazionismo simbolico. Bologna: Il Mulino.

Bortoli, Bruno (2006). I giganti del lavoro sociale. Trento: Erikson.

Bortoli, Bruno (2002). Agli albori della professione del «lavoratore sociale». En C. Marzotto (coord.), Per un'epistemologia del Servizio sociale. La posizione del soggetto (pp. 131149). Milano: Franco Angeli.

Bortoli, Bruno (1997). Teoria e storia del servizio sociale. Roma: Carocci.

Bouquet. Brigitte (2006). Préface. En M. Richmond, Le méthodes nouvelles d'assistance. Le service social des cas individuels, Rennes Cedex: Editions ENSP.

Collins, Randall (1988). Theoretical Sociology. Orlando: Harcourt Brace Jovanovich, Inc. Trad it. 2006: Teorie sociologiche. Il Mulino: Bolonia.

Consiglio Nazionale Ordine Assisitenti Sociali (2009). Codice Dentologico. Roma. Recuperado de: WWW.CNOAS.IT.

Corsini, Mario et al. (1966). Bibliografia italiana di Servizio sociale. Roma: Istituto per gli Studi di Servizio sociale.

Dal Pra Ponticelli, Maria (2010). Nuove prospettive per il sevizio sociale. Roma: Carocci.

Dal Pra Ponticelli, Maria ( 1987). Lineamenti di servizio sociale. Roma: Astrolabio.

Ead (coord.) (2005). Dizionario di Servizio sociale. Roma: Carocci.

Ead (coord.) (1985 a). I modelli teorici del servizi sociale. Roma: Astrolabio.

Ead (coord.) (1985 b). Metodologia del Servizio sociale. Milano: Franco Angeli.

Ead (coord.) (1983). Gli obiettivi del Servizio sociale in rapporto al tema del cambiamento, bisogni e relazione interpersonale. En E. Bianchi et al., Servizio sociale, sociologia, psicologia: ripresa critica di un dibattito teorico (pp. 23-29). Padova: Fondazione Zancan.

Dewey, John (1925). Experience and nature. Chicago: Open Court. Trad. it. 1990: Esperienza e natura. Milano: Mursia.

Diomede Canevini, Milena (1987). Servizio sociale. En F. De Marchi et al., Dizionario di sociologia. Cinisello Balsamo: Edizioni Paoline.

Du Ranquet, Mathilde (1989). Les approches en service social. Canadá: Centurion.

Fargion, Silvia (2009). Il servizio sociale. Storia, temi, dibattiti. Bari: Laterza. 
Ferrario, Franca (2004). Le dimensioni dell'intervento sociale. Un modello unitario centrato sul compito. Roma: Carocci.

Geremek, Bronislaw (1986). La pietà e la forca. Bari: Economica Laterza.

Germain, Carel y Gitterman, Alex (1980). The life model of social work practice: Advances in theory \& practice. Nueva York: Columbia University Press. Trad it. L'intervento del servizio sociale secondo il modello esistenziale. En M. Dal Pra Ponticelli (coord.) (1985 a): I modelli teorici del servizio sociale (pp. 158-193). Roma: Astrolabio.

Gui, Luigi (2004). Le sfide teoriche del servizio sociale. Roma: Carocci.

Leonard, Peter (1966). Sociology in Social Work. Londres: Toutledge \& Kegan. Trad. it.1971: Sociologia e Servizio sociale. Roma: Astrolabio.

Marletti, Carlo (2006). Razionalità e valori. Introduzione alle teorie dell'azione sociale. Bari: Laterza.

Marzotto, Costanza (coord.) (2002). Per un'epistemologia del Servizio sociale. La posizione del soggetto. Milán: Franco Angeli.

Ministero per l'Assistenza post bellica e UNRRA (promotor) (1947). Atti del Convegno di studi di Assistenza sociale. Milán: Marzorati.

Neve, Elisabetta (2008). Il Servizio sociale. Fondamenti e cultura di una professione. Roma: Carocci.

Perlman Harris, Helen (1957). Social case work: A problem solving process. Chicago: University of Chicago Press.

Pincus, Allen y Minahan, Hanna (1977). Work Practice: Divisions and Unifications. En H. Specht y A. Vickery. Londres: Allen \& Unwin. Trad it. 1985: Un modello integrato per la pratica del servizio sociale. En M. Dal Pra Ponticelli (1985 a.), I modelli teorici del servizi sociale (pp. 246-285). Roma: Astrolabio.

Pittaluga, Marisa (2000). L'estraneo di fiducia. Competenze e responsabilità dell'assistente sociale. Roma: Carocci.

Putnam, James J. (1921). Addresses on Psycho-Analysis. Londres: G. Allen \&Unwin. En M. Richmond (1917). Social Diagnosis. Nueva York: Russell Sage Foundation.

Richmond, Mary E. (1917). Social Diagnosis. Nueva York: Russell Sage Foundation.

Richmond, Mary E.(1922). What is Social Case Work? An Introductory Description. Nueva York: Russell Sage Foundation. Trad. francesa 2006: Les méthodes nouvelles d'assistance. Le service social des cas individuals. Rennes Cedex: Editions ENSP.

Sicora, Alessandro (2005). Multidimensionalità. En M. Dal Pra Ponticelli (coord.), Dizionario di servizio sociale, (pp. 392-394). Roma: Carocci.

Tarugi, Paolina (1961). Il Servizio sociale. Milano: UNSAS.

Vallin, Odile (1947). Problemi della formazione tecnica delle assistenti sociali e dell'organizzazione delle scuole di servizio sociale. En Ministero per l'Assistenza post bellica e UNRRA, Atti Convegno di studi di Assistenza sociale (pp. 741- 754). Milán: Marzorati. 